EPJ Web of Conferences 28, 12025 (2012)

DOI: $10.1051 /$ epjconf/20122812025

(C) Owned by the authors, published by EDP Sciences, 2012

\title{
Quarkonium production at ATLAS
}

Darren D. Price ${ }^{\mathrm{a}}$ (on behalf of the ATLAS collaboration)

Department of Physics, Indiana University, Bloomington, IN 47405, USA.

\begin{abstract}
The production of quarkonium is an important testing ground for QCD calculations. The $J / \psi$ and $\Upsilon$ production cross-sections are measured in proton-proton collisions at a centre-of-mass energy of $7 \mathrm{TeV}$ with the ATLAS detector at the LHC. Differential cross-sections are presented as a function of transverse momentum and rapidity. The fraction of $J / \psi$ produced in B-hadron decays is also measured and the differential cross-sections of prompt and non-prompt $J / \psi$ production determined separately. Measurements of the fiducial production crosssection of the $\Upsilon(1 \mathrm{~S})$ and observation of the $\chi_{c, b J}$ states are also discussed.
\end{abstract}

\section{Introduction}

Quarkonia are formed from a quark pair of same flavour and should represent one of the simplest systems described by QCD theory. Heavy quarkonium is a multiscale system, allowing for rigorous tests of the interplay between perturbative and non- perturbative QCD and a rich spectrum of radial and orbital excitations allow studies of spectroscopy and decay dynamics [1]. In addition, quarkonia is an ideal probe of cold and hot nuclear matter effects [2]. Many open questions exist in this area, and it has proven difficult thus far to universally describe the kinematics and production properties of quarkonia. ATLAS plans to investigate quarkonium production through measurements of production, spin-alignment and associated hadronic activity of these various states. The first production measurements toward this goal are outlined below.

\section{Inclusive, prompt and non-prompt $J / \psi$ production cross-section measurements}

Cross-section measurements presented here make use of data collected via a single muon trigger, first with no threshold on $p_{T}$ then later with a threshold at $4 \mathrm{GeV}$ as instantanous luminosity increased. Trigger and reconstruction efficiencies are measured in data and validated with Monte Carlo simulations. Weights incorporating acceptance and efficiency corrections are applied to quarkonia candidates on an event-by-event basis before fits are used to extract a cross-section.

The acceptance corrections represent the probability for quarkonium with given kinematics to pass basic selection cuts. The exact value of this probability in a given phase space is dependent on nature of the as yet unmeasured spin alignment of quarkonium. Five spin-alignment scenarios have been identified that induce the largest envelope of variation on visible cross-sections. Measurements are repeated under application of different acceptance maps reflecting di fferent polarisation states as a systematic effect on measured production observables.

\footnotetext{
a e-mail: Darren.Price@cern.ch
}

Production of $J / \psi$ can occur promptly from the hard interaction, or may be produced non-promptly via decay of a B-hadron. $J / \psi$ from b-decays have positive displaced dimuon vertices and can be distinguished from prompt production (and non- $J / \psi$ backgrounds) via the pseudo-proper time discriminant $\tau=L_{x y} \cdot m_{\mathrm{PDG}}^{J / \psi} / p_{T}^{J / \psi}$, where $L_{x y}$ is the transverse decay length of the $J / \psi$ vertex.

A simultaneous unbinned maximum likelihood fit to invariant mass and lifetime allows us to distinguish prompt and non-prompt $J / \psi$ production from combinatorial background (see Figure 1 for an example of such a fit projected onto the lifetime distribution) and determine the fraction of $J / \psi$ produced via $B$-decays as a function of $p_{T}$ and rapidity (results for one rapidity slice are shown in Figure 2).

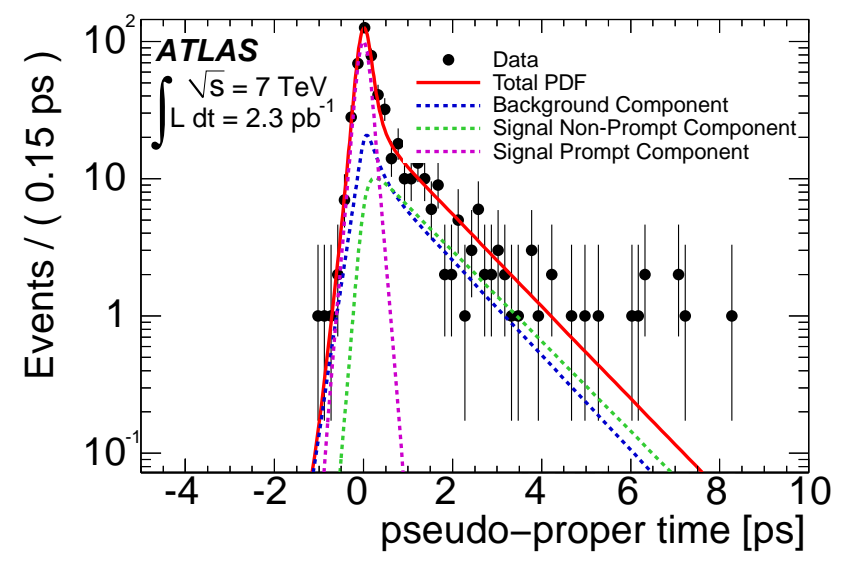

Fig. 1. Pseudo-proper time distribution of $J / \psi \rightarrow \mu^{+} \mu^{-}$candidates in the signal mass region, for $9.5<p_{T}(J / \psi)<10.0 \mathrm{GeV}$ and $\left|y^{J / \psi}\right|<0.75$. The points represent measured data, the solid line is the result of an unbinned maximum likelihood fit to all dimuon pairs in the $2.5-3.5 \mathrm{GeV}$ mass region projected onto the pseudo-proper lifetime distribution.

The data analysed allow for study of the $B$-production fraction across a $p_{T}$ range of 6 to $70 \mathrm{GeV}$, to far higher transverse momenta than have previously been studied. A strong dependence of the fraction is observed as a func- 


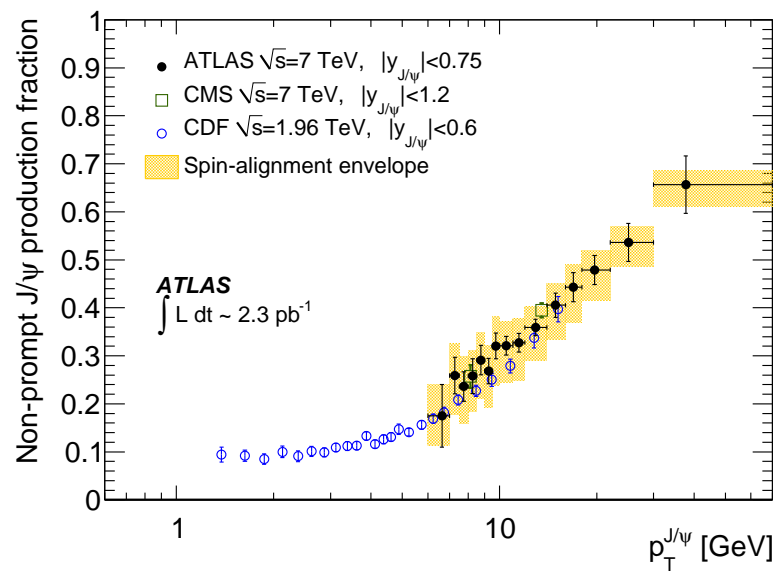

Fig. 2. Production fraction of non-prompt to inclusive $J / \psi$ as a function of $J / \psi$ transverse momentum. Overlaid is a band representing the maximal variation of the result under various spinalignment scenarios. Comparison is made to existing CDF and CMS results.

tion of $p_{T}$, but slow dependence on rapidity in the range $(0<|y|<2.4)$ of rapidities studied. Good agreement with CDF data $(p \bar{p}$ collisions at $\sqrt{s}=1.96 \mathrm{TeV})$ is observed within the experimental uncertainties, suggesting the $B$ fraction has limited dependence on centre-of-mass energy and initial colliding particles, particularly at large $p_{T}$.

Applying candidate-by-candidate efficiency and acceptance weights to di-muon pairs in $p_{T}-y$ bins, extract signal yield from unbinned maximum likelihood fit to $J / \psi$ peak. Figure 3 shows an example of an inclusive differential cross-section extracted for one rapidity bin (four in total), as a function of $J / \psi p_{T}$.

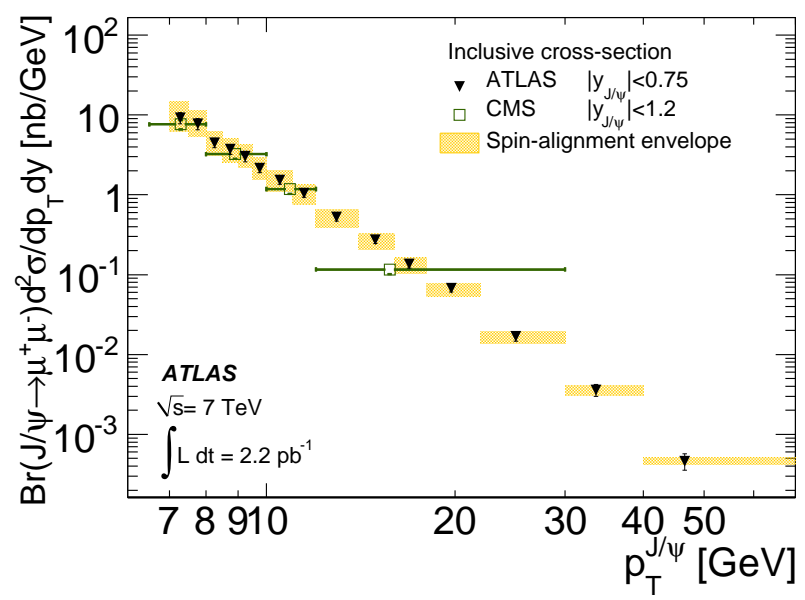

Fig. 3. Inclusive $J / \psi$ production cross-section as a function of $J / \psi$ transverse momentum in the $|y|<0.75$ rapidity bin.

By combining the information from the inclusive crosssection and $B$-fraction measurements, one can extract a non-prompt $(J / \psi$ from $b$-decays) and prompt $J / \psi$ crosssection versus $p_{T}$ and rapidity. Measurements of $J / \psi$ from $b$-decays is found to agree well with Fixed-Order Next-toLeading-Log theoretical predictions. Comparisons to colour singlet NNLO* pQCD predictions and the phenomenological Colour Evaporation Model show significant discrep-
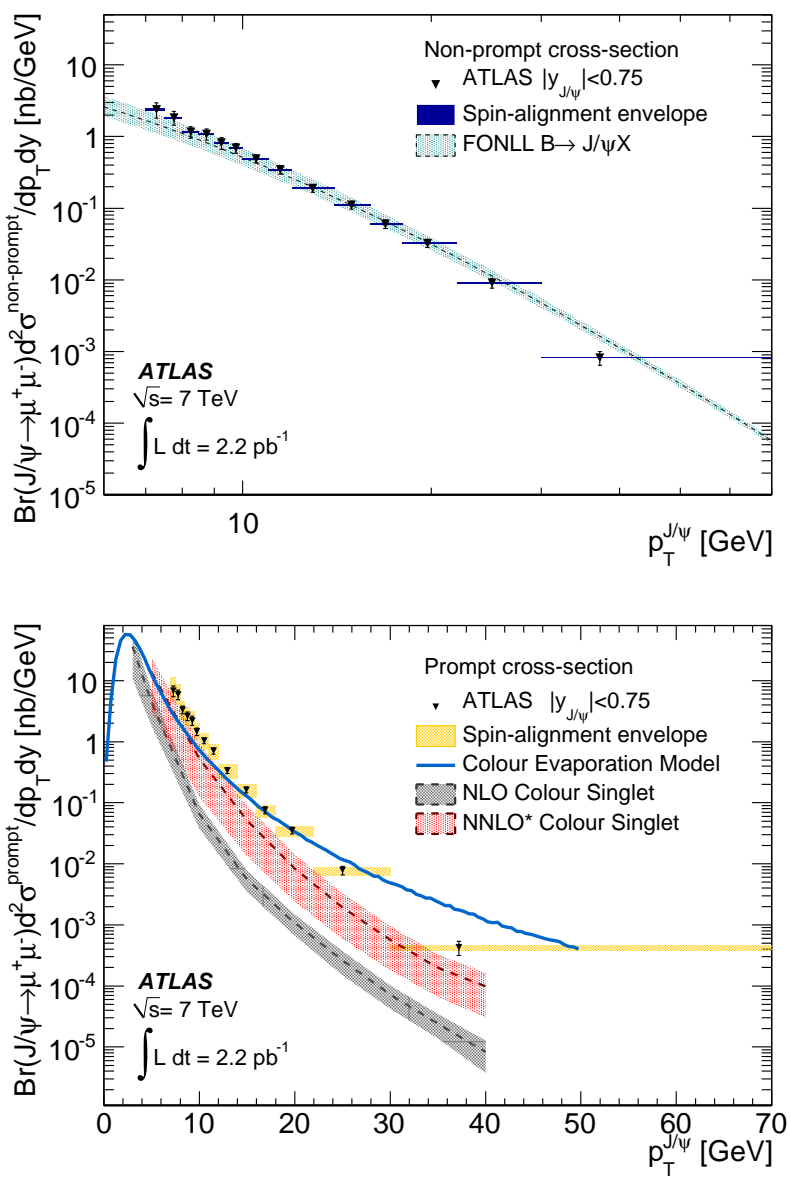

Fig. 4. Non-prompt (top) and prompt (bottom) $J / \psi$ production cross-sections as a function of $J / \psi$ transverse momentum, compared to theoretical predictions (FONLL, in the case of nonprompt production; NLO and NNLO* and Colour Evaporation Model predictions for prompt production). Overlaid is a band representing the variation of the result under various spin-alignment assumptions on the non-prompt and prompt components. The central value assumes an isotropic polarisation for both prompt and non-prompt production.

ancies in shape and normalisation, highlighting the uncertainties that exist in explaining the nature of prompt $J / \psi$ production.

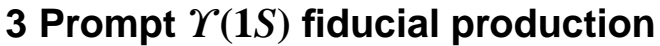 cross-section measurement}

Measurement of the $\Upsilon(1 S)$ cross-section has been conducted in a similar manner as with $J / \psi$, also using single muon triggers. In contrast to the $J / \psi$ results, this measurement is presented in a fiducial region: $p_{T}^{\mu}>4 \mathrm{GeV}$, $\left|\eta^{\mu}\right|<2.5$ to remove the uncertainty due to spin alignment in the cross-section measurement.

Unfolded differential cross-sections are compared to NLO pQCD and significant disagreement is noted. This behaviour is consistent with other theoretical predictions [7] at NLO when compared to prompt $J / \psi$ and $\Upsilon$ data at the Tevatron and LHC, highlighting the need for additional higher order contributions to account for the observed production. 

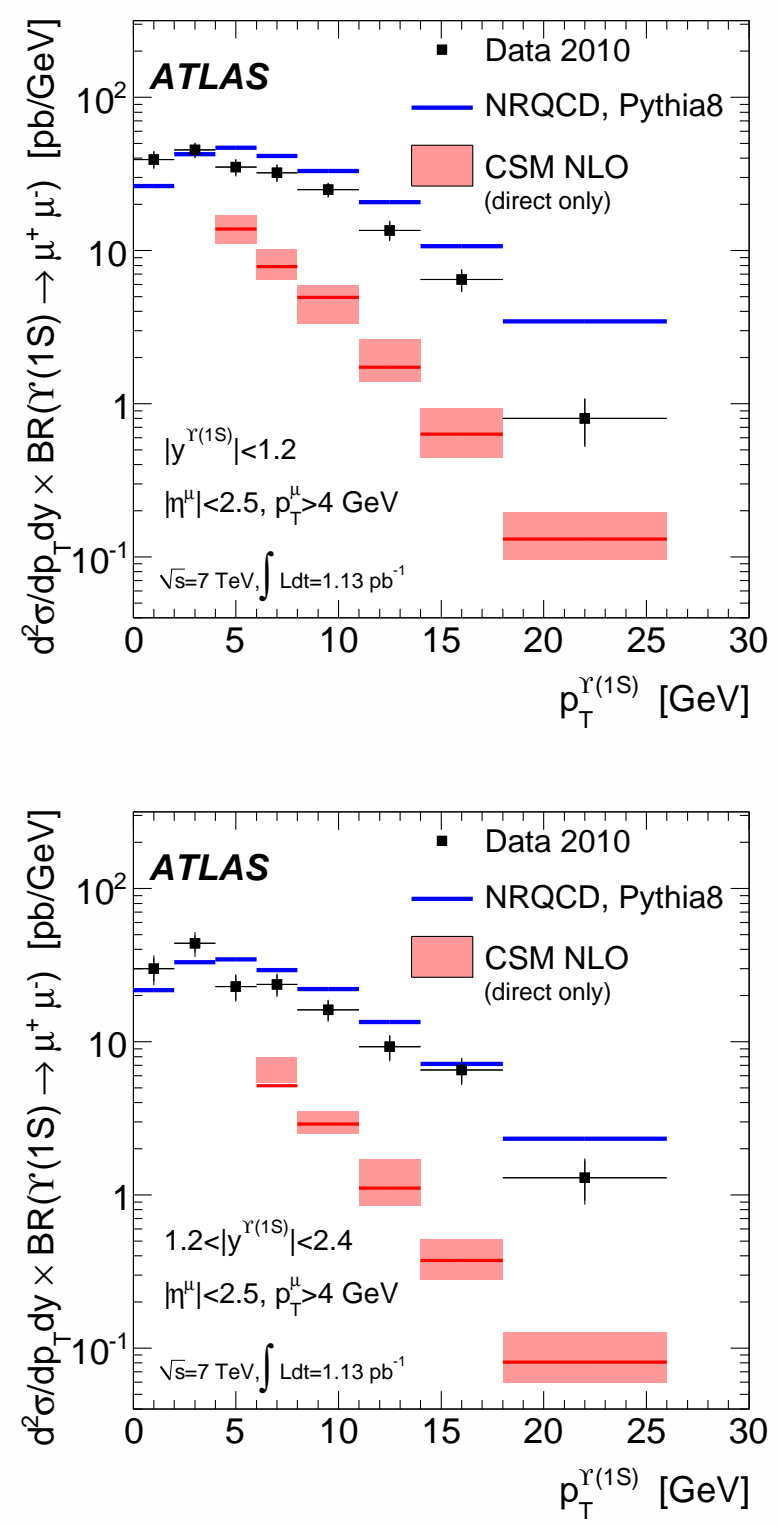

Fig. 5. Differential prompt $\Upsilon(1 S) \rightarrow \mu^{+} \mu^{-}$production crosssection for $\left|y^{\Upsilon(1 S)}\right|<1.2$ (top) and $1.2<\left|y^{\Upsilon(1 S)}\right|<2.4$ (bottom) as function of $p_{T}^{r(1 S)}$ for $p_{T}^{\mu}>4 \mathrm{GeV},\left|\eta^{\mu}\right|<2.5$. Comparison is made to colour-singlet NLO direct production predictions, and the shaded area shows the change in the theoretical prediction when varying the renormalisation and factorisation scales by a factor of two. A comparison is also made to a model based on NRQCD native to PYTHIA8, for a particular choice of parameters.

\section{Reconstruction of $\chi_{c J}$ mesons through radiative decays}

The $\chi_{c 1}$ and $\chi_{c 2}$ mesons in radiative decays to a $J / \psi$ and a photon have been observed via calorimetry. Measuring the production cross-sections of the $\chi_{c J}$ are crucial for precise understanding of $J / \psi$ production and acts as a test of pQCD in its own right.

Photons at ATLAS can also be identified and measured through reconstruction of the tracks from electron-position pairs associated with a photon conversion occurring in the Inner Detector. The tracking capabilities of ATLAS allow for much more precise measurement of the photon kine- matics through this method, but is balanced by a low probability for a conversion to occur and successfully be reconstructed. Measurement via calorimetric techniques (as shown in Figure 6) benefit from increased signal efficiency at a cost of reduced resolution. Recently, similar techniques have been used by ATLAS to reconstruct the $\chi_{b J}(m P)$ states (via $\Upsilon(n S)+\gamma$ decays) in both calorimetry and tracking modes and has led to the discovery [9] of a new set of states, the $\chi_{b J}(3 P)$.

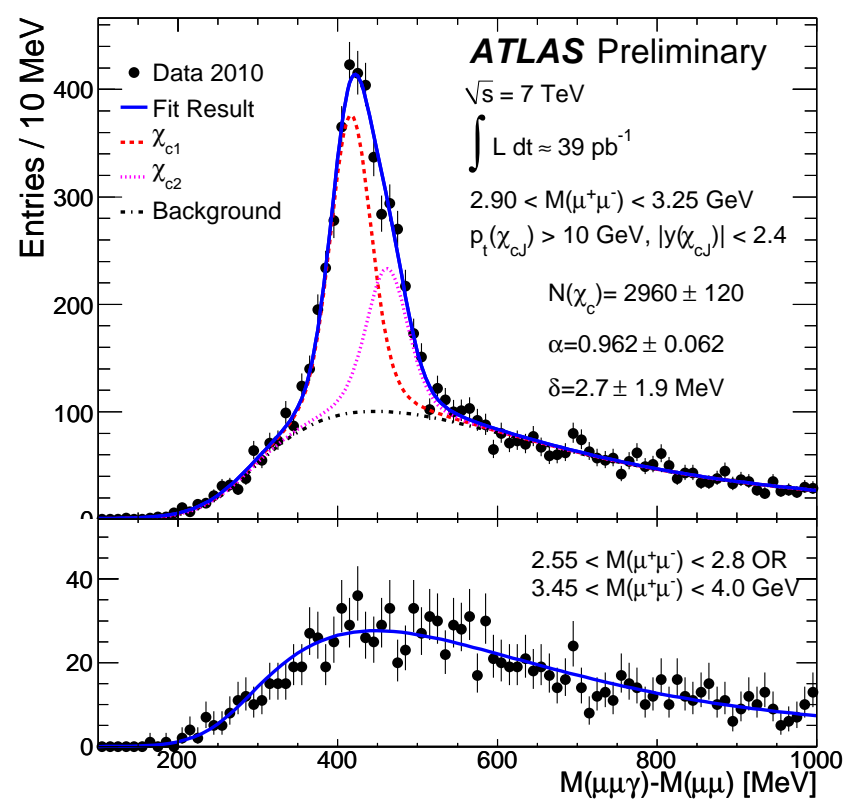

Fig. 6. The results of a simultaneous fit to the signal region (top) and background $(J / \psi$ sideband) region (bottom), showing signals of $\chi_{c 1}$ and $\chi_{c 2}$ mesons reconstructed (via calorimetry measurement) through their radiative decays to $J / \psi+\gamma$, viewed as a function of the invariant mass difference between the $\mu \mu \gamma$ and $\mu \mu$ systems.

\section{References}

1. N. Brambilla et al. [Quarkonium Working Group Collaboration], hep-ph/0412158; N. Brambilla et al., Eur. Phys. J. C 71 (2011) 1534 [arXiv:1010.5827 [hep-ph]].

2. The ATLAS Collaboration, Phys. Lett. B 697 (2011) 294 [arXiv: 1012.5419 [hep-ex]].

3. The ATLAS Collaboration, Nucl. Phys. B 850 (2011) 387 [arXiv:1104.3038 [hep-ex]].

4. D. Acosta et al. [CDF Collaboration], Phys. Rev. D 71 (2005) 032001 [hep-ex/0412071].

5. V. Khachatryan et al. [CMS Collaboration], Eur. Phys. J. C 71 (2011) 1575 [arXiv:1011.4193 [hep-ex]].

6. The ATLAS Collaboration, Phys. Lett. B 705, 9 (2011) [arXiv:1106.5325 [hep-ex]].

7. J. P. Lansberg, Eur. Phys. J. C 61 (2009) 693 [arXiv:0811.4005 [hep-ph]].

8. The ATLAS Collaboration, ATLAS-CONF-2011-136 (https://cdsweb.cern.ch/record/1383839).

9. The ATLAS Collaboration, arXiv:1112.5154 [hep-ex]. 